\title{
Comparative evaluation of group-based mindfulness- based stress reduction and cognitive behavioural therapy for the treatment and management of chronic pain: A systematic review and network meta- analysis
}

\author{
Eve-Ling Khoo, ${ }^{1,2}$ Rebecca Small, ${ }^{1,3}$ Wei Cheng, ${ }^{1}$ Taylor Hatchard, ${ }^{4}$ Brittany Glynn, ${ }^{1}$ \\ Danielle B Rice, ${ }^{1,5}$ Becky Skidmore, ${ }^{6}$ Samantha Kenny, ${ }^{1,7}$ Brian Hutton, ${ }^{1}$ \\ Patricia A Poulin ${ }^{1,8,9}$
}

- Additional material is published online only. To view please visit the journal online (http://dx.doi.org/10.1136/ ebmental-2018-300062).

For numbered affiliations see end of article.

Correspondence to Dr Patricia A Poulin, Ottawa Hospital Research Institute, The Ottawa Hospital, Ottawa, ON K1H 8L6, Canada; ppoulin@ toh.on.ca

Received 13 September 2018 Revised 14 December 2018 Accepted 20 December 2018 Published Online First 23 January 2019

\begin{abstract}
Question This review compares mindfulness-based stress reduction (MBSR) to cognitive-behavioural therapy (CBT) in its ability to improve physical functioning and reduce pain intensity and distress in patients with chronic pain $(C P)$, when evaluated against control conditions.

Study selection and analysis Ovid MEDLINE, EmbaseClassic+Embase, PsycINFO and the Cochrane Library were searched to identify randomised controlled trials. The primary outcome measure was physical functioning. Secondary outcomes were pain intensity and depression symptoms. We used random and fixed effects (RE and FE) network meta-analyses (NMA) to compare MBSR, CBT and control interventions on the standardised mean difference scale.

Findings Twenty-one studies were included: 13 CBT vs control $(n=1095), 7$ MBSR vs control $(n=545)$ and 1 MBSR vs CBT vs control $(n=341)$. Of the 21 articles, 12 were determined to be of fair or good quality. Findings from RE NMA for change in physical functioning, pain intensity and depression revealed clinically important advantages relative to control for MBSR and CBT, but no evidence of an important difference between MBSR and CBT was found.

Conclusions This review suggests that MBSR offers another potentially helpful intervention for CP management. Additional research using consistent measures is required to guide decisions about providing CBT or MBSR.
\end{abstract}

\section{BACKGROUND}

Chronic pain (CP) affects one in five adults and may impact all dimensions of a person's well-being. ${ }^{1}$ The prevailing psychological intervention for CP is cognitive-behavioural therapy (CBT). ${ }^{23} \mathrm{CBT}$ focuses on increasing flexibility in thoughts and behaviours to respond more adaptively to challenges. In the context of CP, CBT often includes psychoeducation about pain, cognitive restructuring of maladaptive pain-related beliefs, problem-solving, relaxation training, behavioural activation and pacing. ${ }^{23}$

Another promising intervention for $\mathrm{CP}$ is mindfulness-based stress reduction (MBSR). MBSR is a groupbased intervention that focuses on building awareness and acceptance of moment-to-moment experiences, including physical discomfort and difficult emotions. ${ }^{4}$ Core components of MBSR include increasing awareness of one's body, emotions, sensations, thoughts as well as learning self-regulation strategies and more adaptive responses to stress.

This systematic review of randomised controlled trials was performed to assess the efficacy of MBSR compared with CBT for CP and pain-related symptoms in adults. At the time of protocol development, ${ }^{5}$ we anticipated that there would be few head-to-head trials comparing these therapies. We therefore planned to use network meta-analysis (NMA) methods ${ }^{67}$ to inform an indirect comparison between interventions, considering standard care (SC) as the most commonly used control group. Efficacy was assessed based on criteria described in the Initiatives on Methods, and Pain Assessment in Clinical Trials (IMMPACT) recommendations. ${ }^{8}$

\section{STUDY SELECTION AND ANALYSIS}

\section{Protocol and registration}

The protocol for this systematic review was published, ${ }^{5}$ registered within the PROSPERO database (CRD42014009356) and follows the Preferred Reporting Items for Systematic reviews and Meta-Analyses extension statement for network meta-analyses (NMA) (see eTable 1 in the online supplementary material). ${ }^{9}$

\section{Study eligibility criteria}

Following the Population-Intervention and Comparators-Outcomes-Study design framework, eligible studies must have been published in English or French in addition to meeting the following criteria:

1. Population: Participants had to be at least 18 years of age, with a minimum three month history of $\mathrm{CP} .^{10}$ This included a wide range of medical profiles (eg, rheumatoid arthritis, back pain, neck pain). Justification for a heterogeneous sample is supported by research detailing the effectiveness and applicability of both CBT and MBSR in a variety of pain conditions. ${ }^{2-4} 11$ 


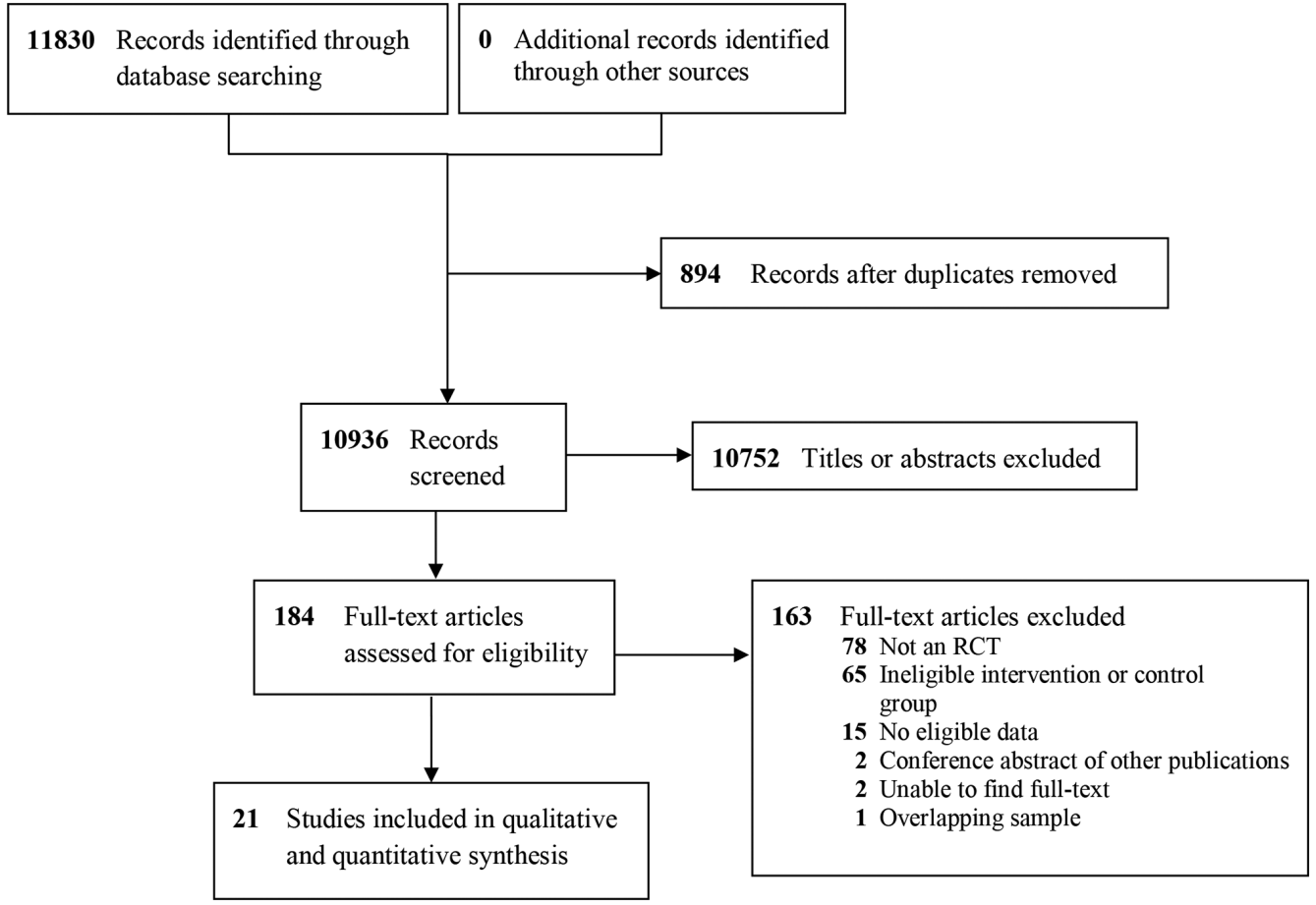

Figure 1 Study selection. RCT, randomised controlled trial.

2. Interventions/Comparators: Studies that investigated the efficacy of CBT, MBSR or both in comparison to SC, passive education, wait-list (WL)/no treatment or the same adjunctive treatment (eg, pharmacotherapy) groups were included. To be eligible, MBSR programmes were required to have 8 to 12 weekly sessions of approximately 2 to 2.5 hours each, include a full-day intensive session and 45 minutes of daily home practice. ${ }^{4}$ Studies with interventions involving other types of mindfulness-based programmes were excluded. CBT programmes had to be group-based, in-person and include 8 to 12 sessions with a minimum of 15 hours in total (see eAppendix 1 for protocol deviations in the online supplementary material). ${ }^{12}$

3. Outcome measures: Based on the IMMPACT recommendations, ${ }^{8}$ outcome measures of interest were physical functioning, pain intensity, emotional functioning and patient's global impression of change (PGIC). Studies were required to report at least one of these outcomes before and after treatment (within two weeks of start and end date). For this review, emotional functioning was restricted to depressive symptoms. Additional details regarding outcome measures of interest are provided in eAppendix 2 in the supplement.

4. Types of studies: Only randomised controlled trials were included.

\section{Information sources and literature search}

Studies were identified through structured searches of electronic databases including Ovid MEDLINE, Ovid MEDLINE In-Process \& Other Non-Indexed Citations, Embase Classic+Embase, PsycINFO and the Cochrane Library. The search strategies were developed and tested through an iterative process by an experienced medical information specialist in consultation with three content experts. The strategies used controlled vocabulary and free-text terms to identify studies pertaining to CP combined with the interventions of interest. Searches were executed on 4 April 2014 and updated on 12 October 2017. No date or language restrictions were employed. Grey literature searches were not performed. Manual review of the bibliographies of included studies was performed to ensure no relevant studies were missed. See eAppendixes 3 and 4 in the online supplementary data for the detailed search strategy.

\section{Process of study selection}

Study selection was conducted in duplicate by eight independent evaluators paired into teams. Evaluators trained, didactically and through pilot screening, on the purpose of the study, treatments being investigated, inclusion and exclusion criteria and outcomes of interest. We first screened study title and abstracts using liberal acceleration, followed by full-text screening. During full-text screening, disagreements among the evaluators were resolved through consensus (see figure 1 for a summary of the study selection process).

\section{Data collection process and details}

Data extraction was performed using a standardised data extraction form in Microsoft Excel (V.2016, Microsoft, Seattle, USA). Two team members independently extracted data for all primary and secondary outcomes as well as a detailed description of the interventions, study design and population characteristics. Discrepancies were resolved by a third evaluator through discussions.

\section{Risk of bias assessment}

The methodological quality of included trials was measured through a risk of bias assessment conducted independently by two evaluators using the US Preventative Services Task Force Quality Rating Criteria ${ }^{13}$ scale (see eAppendix 1 in the online supplementary material). Disagreements were discussed; if consensus was not achieved, a third assessor resolved the disagreement. 


\section{Synthesis of evidence}

Network diagrams were prepared to study the patterns of comparisons and outcome data for each endpoint. These figures convey the amount of information available for each analysis in terms of numbers of studies assessed per treatment and numbers of studies supporting each comparison in the network. The primary analysis for this review focused on comparisons between three groups: CBT, MBSR and control. Bayesian NMA of standardised mean differences (SMD) were performed to obtain summary measures of effect size across studies to quantify the impact of treatment relative to control. This enabled synthesis of data measuring the same outcomes using different scales. Included studies reported their findings using different approaches; some reported mean changes from baseline with corresponding standard errors (SEs), while others reported only mean values at baseline and post-treatment with corresponding standard deviations (SDs) for each treatment arm. We converted data in one study ${ }^{14}$ to the former scenario to use in NMA, assuming the variance of the change per arm was the same (see eAppendix 5 in the online supplementary material). For the latter scenario, we placed a prior distribution (Uniform $(0.5,1))$ on the correlation between mean values at baseline and follow-up and assumed the correlation to be common across interventions and studies and calculated the mean changes from baseline and corresponding SEs for both arms when they were not reported. We modified a well-established mode $^{15}$ with a normal likelihood and identity link to perform NMAs on the SMD scale using the mean and variance of change from baseline per arm as inputs. The mean difference between any two treatment arms was rescaled by a pooled within-study SD (not depending on the SMD or mean difference) for standardisation. Comparisons of the effects of interventions for each outcome of interest were estimated as SMDs (Hedges' g) along with 95\% credible intervals.

Random and fixed effects (RE and FE) Bayesian NMAs were run to obtain summary estimates for the treatment effects. Posterior median SMDs were collected for all pairwise comparisons along with corresponding 95\% credible intervals; for the purposes of interpretation, SMDs of $0.2-0.5,0.5-0.8$ and $>0.8$ were considered to represent small, moderate and large effect sizes, respectively. ${ }^{16}$ Secondary measures of effect including Surface Under the Cumulative Ranking Curve (SUCRA, ranging from 0 to 1 , with values nearest 1 indicating preferred treatments), mean treatment rankings and 'probability better' values (ie, the pairwise probability that an intervention is better than another) were also calculated. Both RE consistency models and RE unrelated means models for NMA were run to monitor for any violations of the consistency assumption between direct and indirect evidence. ${ }^{15}$

All analyses were run using 100000 burn-in iterations and 100000 sampling iterations. Model fitting was assessed by comparing total residual deviance and deviance information criteria (DIC) across models. Selection between models was based on comparison of DIC, with a threshold of five points or more (with lower values preferred) indicative of an important difference in fit; findings from RE consistency models were preferred unless measures of model fit were found to greatly favour those from the RE unrelated means model or the FE consistency model. All NMAs were performed using OpenBUGS software V.3.2.3 $3^{17}$ and R2OpenBUGS software V.3.2-3.2 ${ }^{18}$ in $\mathrm{R}$ (see eAppendix 6 in the online supplementary material 1 for the $\mathrm{R}$ Codes to Call OpenBUGS used for the NMA). Model convergence was assessed using established methods including
Gelman-Rubin diagnostics and the Potential Scale Reduction Factor (Rhat). ${ }^{17}$

We used the RE meta-analysis model (DerSimonian-Laird) ${ }^{19}$ for pairwise meta-analyses on the SMD scale for each outcome (see eAppendix 7 in the online supplementary material 1). For studies in which only the preintervention and postintervention mean and SD per arm were available, we assumed that the correlation between preintervention and postintervention scores to be fixed at the posterior mean estimate of the correlation from NMA (RE consistency model).

In addition to the primary analyses focused on comparisons between CBT, MBSR and control, in sensitivity analyses we also considered an alternative network geometry where the representation of control therapies was further refined. Analogous methods were used for all sensitivity analyses.

\section{FINDINGS \\ Study selection, characteristics and results of individual studies}

A total of 10936 unique records were identified. Screening of titles and abstracts excluded 10752 irrelevant records, leaving 184 full-text articles for full-text screening. Full-text screening excluded an additional 163 articles (see eTable 2 for excluded articles and reasons), leaving a total of 21 publications describing 21 studies for the review. ${ }^{14-39}$ An overview of study characteristics is provided in table 1 .

\section{Overview of participant characteristics}

There were a total of 1981 participants. Participants were predominantly women $(81 \%$ of studies reporting $60 \%$ or more women) with an average age between 35 and 65 years old. While 12 studies $(52 \%)$ did not provide any information regarding participant ethnicity, $142122262729-333739$ nine (43\%) reported greater than $60 \%$ of participants as being Caucasian..$^{2023-2534-3638}$

Most studies focused on musculoskeletal pain, where nine studies (43\%) focused on fibromyalgia, ${ }^{20} 23272831-333637$ three on chronic low back pain (14\%), ${ }^{14} 3034$ two on rheumatoid arthritis, ${ }^{21} 25$ one on osteoarthritis of the knee, ${ }^{39}$ temporomandibular disorder, ${ }^{24}$ failed back surgery syndrome ${ }^{35}$ and Gulf War illness, respectively. ${ }^{38}$ One study had a mixture of musculoskeletal pain conditions ${ }^{29}$ and the remaining two studies did not specify the CP conditions included. ${ }^{22} 26$ Eight studies (38\%) reported participants with an average pain duration of more than ten years; 2021262732333739 nine (43\%) had participants with an average pain duration of between 3 and 9 years. ${ }^{1422} 242528-303436$ Four (19\%) did not provide this information. ${ }^{23} 313538$

\section{Overview of interventions and endpoints reported}

Figure 2, panels A-C present the evidence networks corresponding to the analyses of physical functioning, pain intensity and depression, respectively. Of the 21 studies, 13 evaluated the effects of CBT 14202123242629303233363739 and seven investigated the effects of MBSR; 22252728313538 one recent study directly compared MBSR to CBT and control. ${ }^{34}$ Minor deviations from the standard CBT or MBSR format were accepted after review and discussion among the research team. Among the 21 studies, 13 used a SC control group, ${ }^{14}$ 20-22 $242532-363839$ six had a WL control group ${ }^{26-28303137}$ and two used an attention control (AC) group. ${ }^{2329}$ Four studies evaluating CBT did not discuss therapist training, ${ }^{29} 333639$ while the remaining studies had interventions administered by psychologists, physicians or social workers trained in CBT as well as supervised psychology students. Although one MBSR study did not provide therapist training 


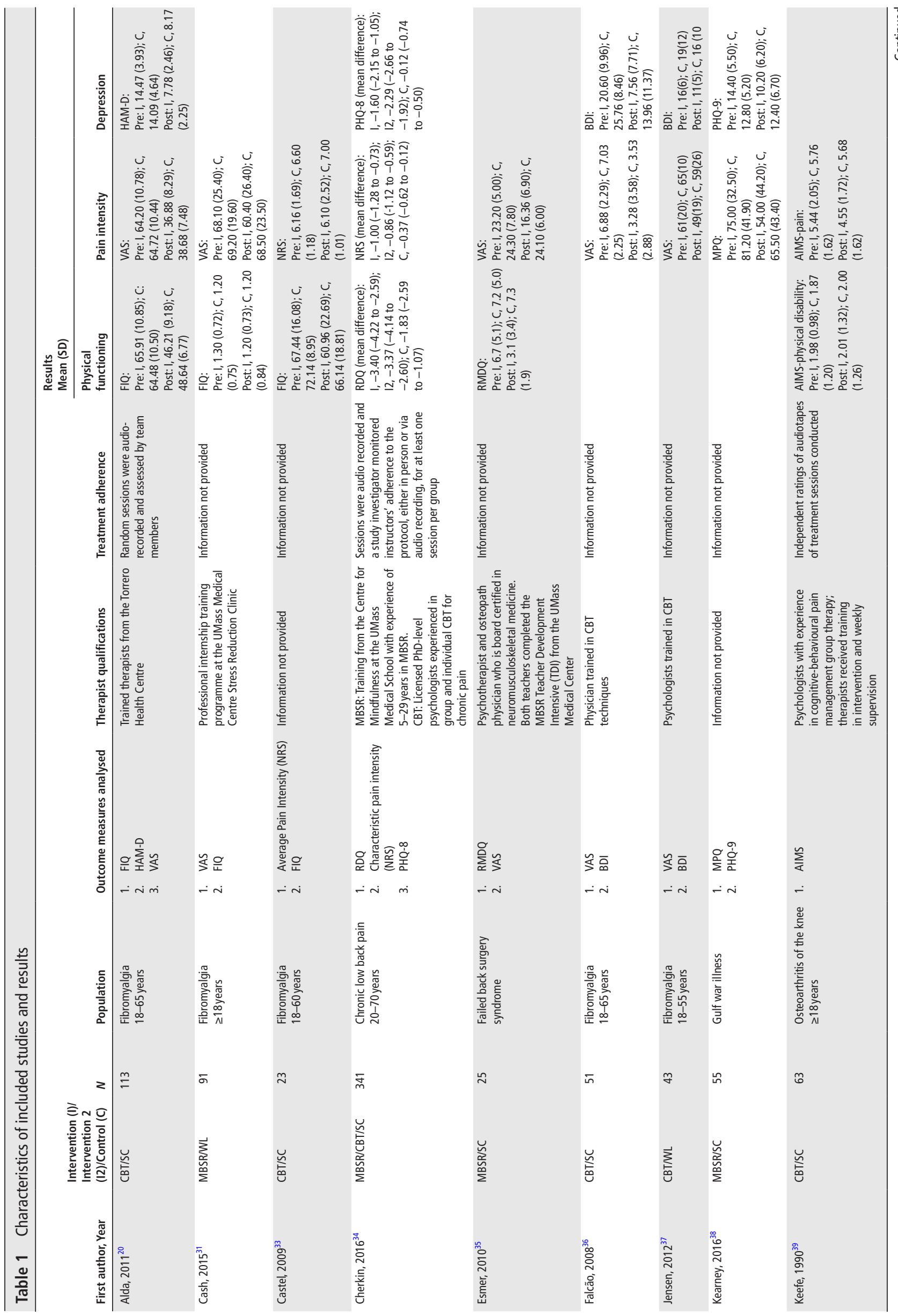




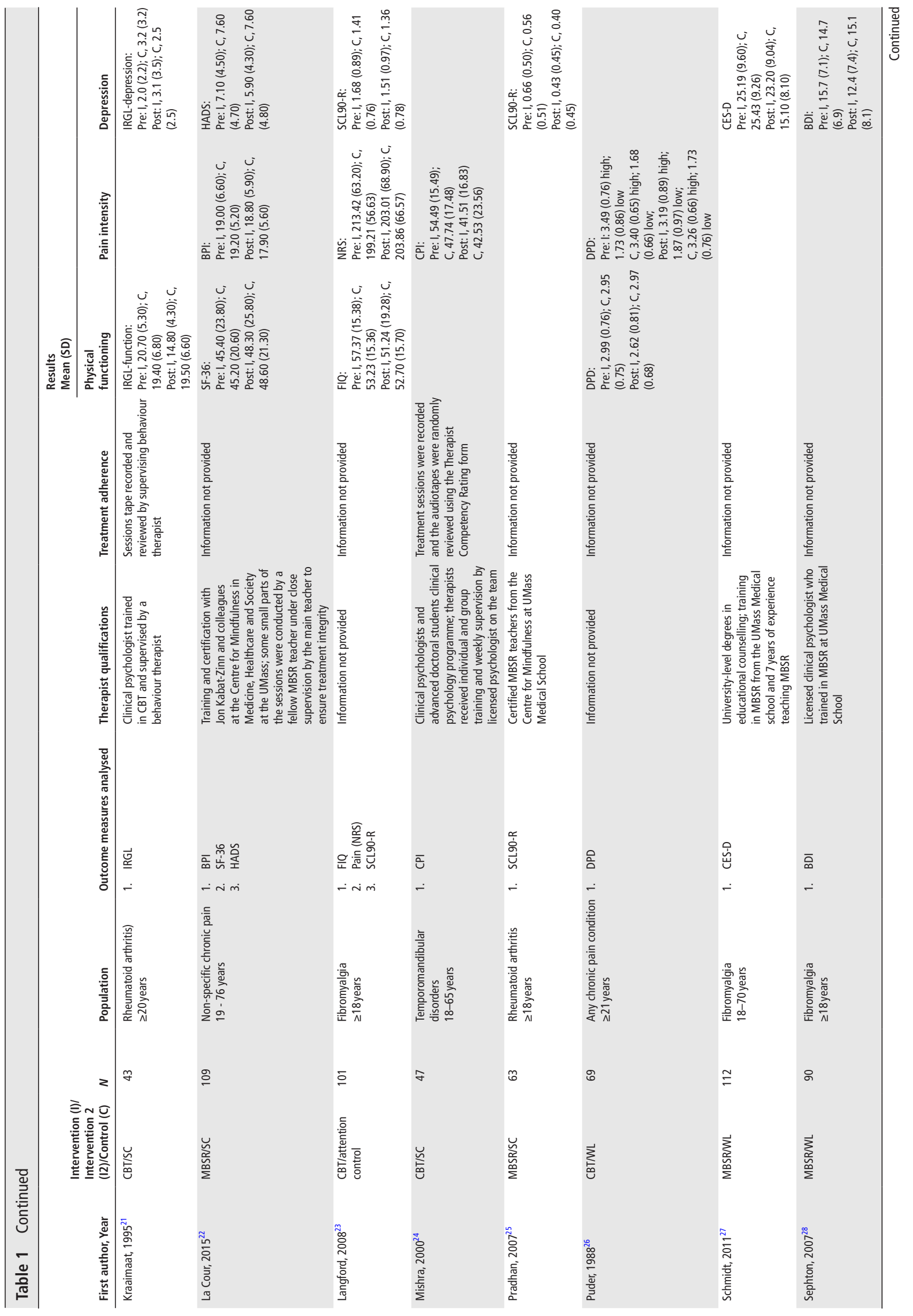

䍗. 
information, the rest employed instructors participated in MBSR teacher training or certification courses at UMass Medical Centre. ${ }^{22} 2527283135$ The MBSR interventions were administered by certified MBSR teachers, ${ }^{22} 2531$ a psychotherapist and osteopath physicians, ${ }^{35}$ education counsellors, ${ }^{27}$ and a psychologist. ${ }^{28}$ Treatment adherence information was not provided in any of the MBSR studies. One study directly compared MBSR to CBT and a SC control group ${ }^{34}$ and involved MBSR instructors who received training from the Centre for Mindfulness at UMass Medical School or had 5-29 years of MBSR experience, while the CBT instructors were licensed psychologists.

Of all studies included, $15(71 \%)$ measured the differences in physical functioning, ${ }^{14}$ 20-23 2627 29-3539 17 studies (81\%) measured pain intensity ${ }^{14} \quad 20-24 \quad 26 \quad 29-31 \quad 33-39$ and one-third measured symptoms of depression. ${ }^{14} 20-232527-29323436-38$ Only one study had measured PGIC resulting in not enough data to analyse this outcome. ${ }^{34}$ Time points were the same for all studies where the baseline or pretreatment was within two weeks of treatment start date and post-treatment was within two weeks of the last treatment date.

\section{Risk of bias within studies}

The quality assessment of included studies is provided in eTable 3 in the online supplementary material. Only four studies received ratings of good quality. ${ }^{28} 313438$ The main reasons for poorer ratings were: lack of intention to treat analyses, ${ }^{24293033353739}$ significant withdrawal rate or absence of attention to withdrawal2 $2-25353739$ and use of measures with unclear validity. ${ }^{14} 20212325-273033353639$ All 21 studies defined interventions clearly, which included content, number of hours and sessions. Important outcomes considered were based on adherence to the IMMPACT guidelines; ${ }^{8}$ studies with at least two outcomes were rated as good $(86 \%)^{1420-242629-39}$ and those reporting one were rated fair (14\%). ${ }^{25} 2728$

\section{Findings, change in physical functioning, pain intensity and depression symptoms}

Model fit statistics for RE NMAs (consistency and unrelated means models) were found to be adequate and comparable to each other for all three endpoints based on comparison of total residual deviance with the total number of unconstrained data points. These values are provided in figure 3 and eTable 4 in the online supplementary material along with corresponding measures for FE consistency models where fit was found to be limited; inspection of DIC between models for all analyses consistently suggested the fit of RE consistency models was preferred. These tables also provide estimates of the between-study SD for all analyses. Findings from RE consistency model analysis identified differences of small effect size for change in physical functioning (SMD of -0.42 , $95 \%$ CrI -0.94 to -0.10 ), pain intensity (SMD -0.34 , $95 \% \mathrm{CrI}-0.79$ to 0.03 ) and depression symptoms (SMD of $-0.49,95 \% \mathrm{CrI}-1.89$ to -0.10 ) that favoured MBSR over control. Comparisons between CBT and control demonstrated differences of small effect size for change in physical functioning (SMD of $-0.40,95 \% \mathrm{CrI}-0.82$ to -0.15 ), pain intensity (SMD $-0.37,95 \% \mathrm{CrI}-0.68$ to -0.13 ) and depression symptoms (SMD of $-0.44,95 \% \mathrm{CrI}-1.29$ to -0.08 ) in favour of CBT. The comparison between MBSR and CBT provided no evidence of an important difference for any of physical functioning (SMD $-0.02,95 \% \mathrm{CrI}-0.49$ to 0.42 ), pain intensity (SMD $0.02,95 \% \mathrm{CrI}-0.43$ to 0.48 ) or depression symptoms (SMD $-0.06,95 \% \mathrm{CrI}-1.08$ to 0.47 ). eTable 
(A) Change in Physical Functioning

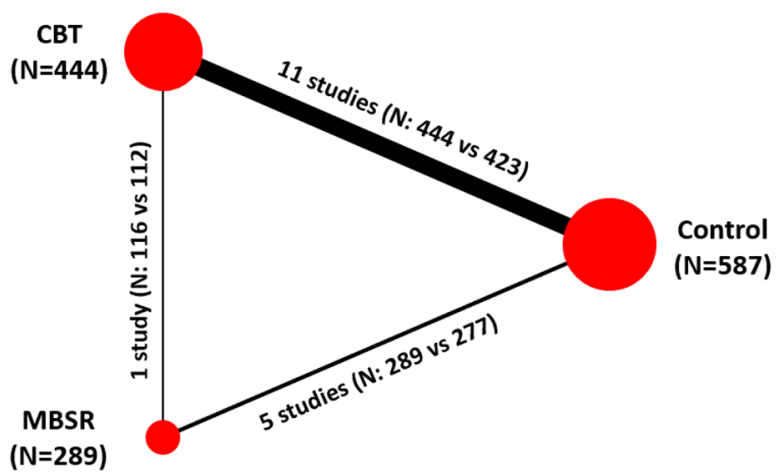

(B) Change in Pain Intensity

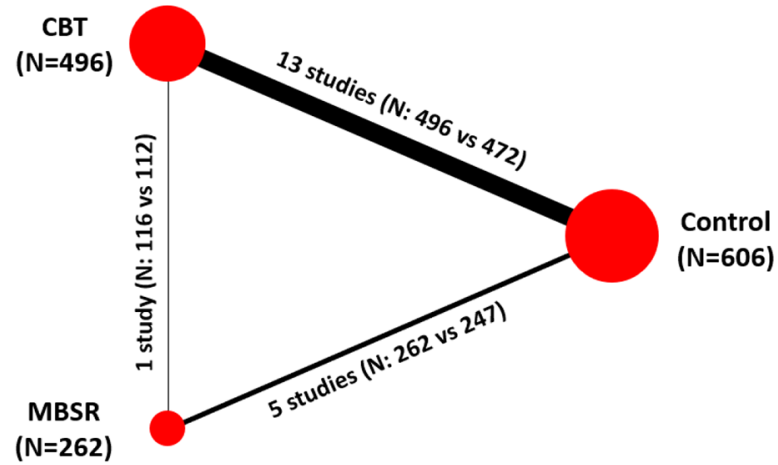

(C) Change in Depression

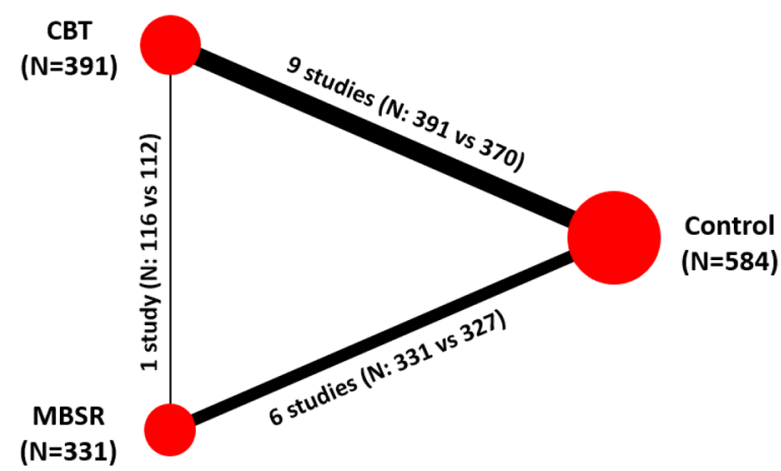

Figure 2 Network diagrams by endpoint. Panels (A)-(C) present networks diagrams of the evidence analysed for the three endpoints. Treatment nodes for each network were sized to proportionately reflect the numbers of patients randomised to each intervention included in the network, while the thickness of the edges joining the treatment nodes was sized to proportionately reflect the number of studies informing each treatment comparison. Note: One study was a three-armed study comparing MBSR $(n=116)$ and $C B T(n=112)$ and control $(n=113){ }^{34} C B T$, cognitive behavioural therapy; MBSR, mindfulness-based stress reduction.

Treatment 1 vs Treatment 2

Physical Functioni
Control vs MBSR
Control vs CBT
CBT vs MBSR
Pain Intensity
Control vs MBSR
Control vs CBT
CBT vs MBSR
Depression
Control vs MBSR
Control vs CBT
CBT vs MBSR

SMD $(95 \% \mathrm{CrI})$

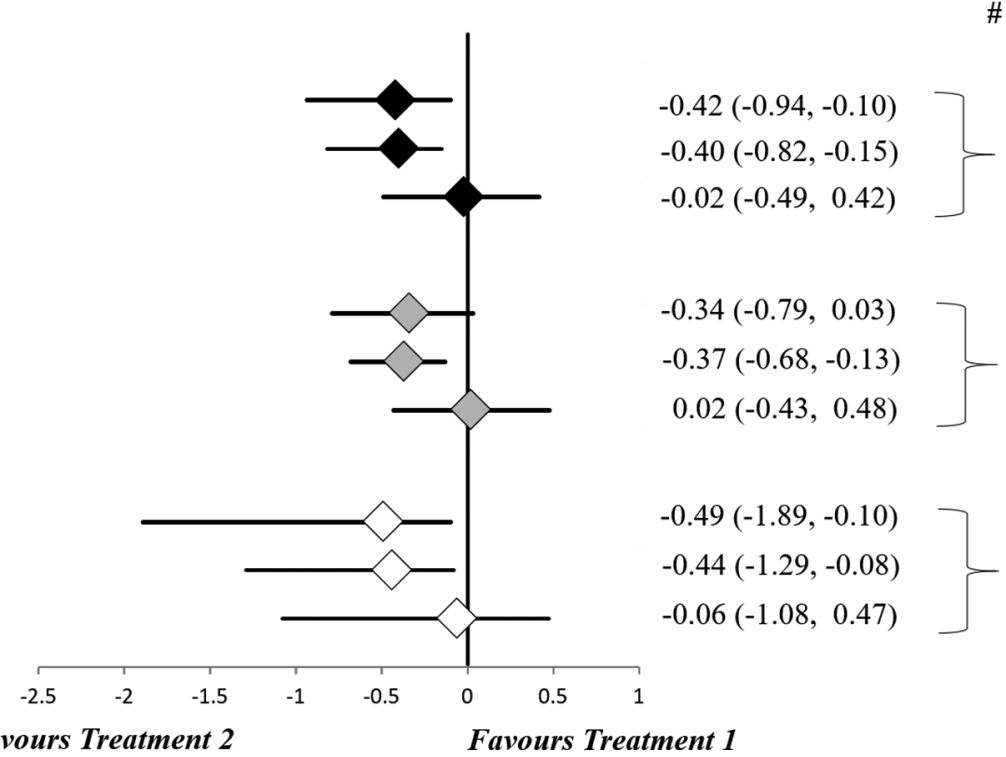

Total Residual Deviance; \# unconstrained data points

Figure 3 Summary of findings from RE NMA-SMD with 95\% Crl. *Number of unconstrained data points=number of arms with reported mean change+number of arms with reported preintervention and postintervention data. CBT, cognitive behavioral therapy; $\mathrm{Crl}$, credible interval; MBSR, mindfulness-based stress reduction; SMD, standardised mean difference. 
5 in the online supplementary material provides league table summaries of all pairwise comparisons of the change in each outcome. Additionally, eTable 6 presents a summary of the secondary effect measures (ie, SUCRA and mean treatment ranks) for each endpoint. The comparison adjusted funnel plots based on Hedges' g and its SE were applied to assess for small-study effects (eAppendix 8) and provided evidence of minimal asymmetry.

\section{Additional analyses}

We also performed sensitivity analyses for each outcome measure where the control group was subdivided into more granular categories: SC and WL. The control groups in two studies received weekly attention-control phone calls ${ }^{23}$ and mailings of education materials, ${ }^{29}$ respectively, which were considered AC. AC was compared with only CBT and was indirectly connected to the rest of the evidence network through CBT, thus these two studies were excluded from sensitivity analyses.

Findings from the sensitivity analyses are presented in detail in eAppendix 9 in the online supplementary data. Results from NMAs demonstrated that WL was not different from SC for changes in physical function, pain intensity and depression symptoms. Having subdivided control into SC and WL, all 95\% credible intervals had widened as a consequence, which obscured the certainty of the moderate-sized SMD estimates of MBSR and CBT versus WL for changes in physical functioning and depression symptoms.

\section{CONCLUSIONS AND CLINICAL IMPLICATIONS}

This systematic review was performed to assess the efficacy of MBSR compared with CBT to treat CP and pain-related symptoms in adults. Thirteen studies compared CBT to control

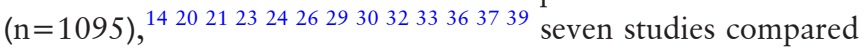
MBSR to control $(\mathrm{n}=545)^{22252728313538}$ and one study compared MBSR to CBT and control $(\mathrm{n}=341) .{ }^{34}$

Point estimates derived from NMAs in the current review were of comparable magnitude for CBT and MBSR, however 95\% credible intervals associated with comparisons between these therapies were found to be very wide. Given this uncertainty and the paucity of head-to-head trials comparing them (only one study ${ }^{34}$ ), it is not yet possible to determine which intervention might be more beneficial for patients with different pain, medical and psychosocial presentations.

The only head-to-head trial that compared MBSR to $\mathrm{CBT}^{34}$ did not find significant differences in improvements for physical functioning and pain intensity. The CBT group, however, did result in significantly more improvement on depression symptoms postintervention compared with the MBSR group. ${ }^{34}$ In the current review, compared with control, we found that both MBSR and CBT were associated with improvements for all three outcome measures. These results were consistent with findings from the head-to-head trial of MBSR, CBT and control. ${ }^{34}$

This also aligns with a meta-analysis from 2016 on acceptance and mindfulness-based interventions for $\mathrm{CP}$ that showed small effects for pain intensity, pain disability and depression and moderate effects for pain interference postintervention compared with control, SC and education groups. ${ }^{40}$ Compared with the current review, the differences in effect sizes may be attributed outcome details as we did not differentiate between pain disability and pain interference as the 2016 review did. Similarly, in a psychotherapy meta-analysis for CP adults, Williams et $a l^{41}$ found CBT to have small to moderate effects on disability, pain intensity and mood postintervention compared with SC or WL groups.

\section{Limitations and recommendations}

While findings from this review support that both MBSR and CBT show benefits related to physical functioning, pain intensity and depressive symptoms for patients with $\mathrm{CP}$, the results should be interpreted carefully; only Cherkin et al ${ }^{34}$ directly compared MBSR and CBT, and there were considerably fewer MBSR studies compared with CBT, resulting in smaller MBSR sample sizes and potentially limiting the reliability of the results. While estimated differences between these therapies approached 0 , they were associated with wide $95 \%$ credible intervals. Additional studies directly comparing MBSR and CBT are needed.

Findings from quality assessments in this review suggest that more efforts are needed during the design stage to enhance the rigour of future studies, including the use of standardised measures and standard follow-up time points (eg, up to 12 months).

The extent of uncertainty in our findings can be partially attributed to the fact that most of the included studies had reported outcomes in terms of mean values at baseline and post-treatment with corresponding SDs, while few studies reported mean changes from baseline with corresponding SEs for each treatment arm. To address this difference, we assumed a prior distribution on the correlation between mean values at baseline and follow-up to calculate SEs of the mean changes from baseline, which was an indispensable step to completing analyses but introduced more uncertainty.

Several forms of clinical and methodological heterogeneity were noted among included studies. Although 15 studies (71\%) had an additional follow-up, they ranged from 8 to 52 weeks postintervention. We recommend a consensus be established on the timing of follow-up to determine whether the benefits of the interventions are sustained over time. Having a heterogeneous group of outcome measures and time points complicates the pooling of data and makes comparison among studies more difficult and potentially less meaningful to patients, clinicians, researchers and decision makers. This heterogeneity may also be a contributing factor to the varied treatment effects found across the studies in this meta-analysis. Additionally, the variability in diagnoses of $\mathrm{CP}$ and the range of therapist qualifications and participant demographics represent other sources of betweenstudy variability.

Another limitation was the lack of intention-to-treat analyses in nearly half the studies in this review (48\%). ${ }^{21} 242629303335-3739$ Also, there were no subgroup analyses to see if participants with different characteristics responded differently. Determining if certain patient characteristics predict response to CBT or MBSR is important to inform clinical guidelines.

Potential outcome differences related to therapist training and treatment adherence could not be assessed given lack of reporting. This is important to guide future research and treatment delivery. For example, therapist adherence to treatment delivery is strongly related to participant symptom changes within treatment protocols; ${ }^{42}$ treatment integrity ${ }^{43}{ }^{44}$ and a positive predictor of therapeutic alliance. ${ }^{45}$

To conclude, while CBT is considered to be the preferred psychological intervention of $\mathrm{CP}$, not all patients with $\mathrm{CP}$ experience a clinically significant treatment response. ${ }^{2}$ Although a number of recommendations have been proposed to improve CBT for patients with $\mathrm{CP}^{46}$ an additional solution may be to offer patients MBSR since it shows promise in improving pain severity and reducing pain interference and psychological distress. 
Additional and more rigorous research that compares CBT and MBSR directly and includes more information about patient characteristics, therapist training and treatment adherence is needed to draw definitive conclusions to inform guidelines.

\section{Author affiliations}

Clinical Epidemiology, Ottawa Hospital Research Institute, Ottawa, Ontario, Canada ${ }^{2}$ School of Rehabilitation Science, McMaster University, Hamilton, Ontario, Canada ${ }^{3}$ Faculty of Medicine, Memorial University of Newfoundland, Newfoundland and Labrador, Canada

${ }^{4}$ School of Psychology, University of Ottawa, Ottawa, Ontario, Canada

${ }^{5}$ Department of Psychology, McGill University, Montreal, Canada

${ }^{6}$ Independent Information Specialist Consultant, Ottawa, Ontario, Canada

${ }^{7}$ Department of Educational \& Counselling Psychology, McGill University, Montreal, Quebec, Canada

${ }^{8}$ Department of Psychology, The Ottawa Hospital, Ottawa, Ontario, Canada ${ }^{9}$ Department of Anesthesia and Pain Medicine, Faculty of Medicine, University of Ottawa, Ottawa, Ontario, Canada

Acknowledgements This review would like to acknowledge: Howard Nathan, Catherine E Smyth, Ola Mioduszewski, Heather Romanow, Erin O'Farrell, Carley Chiasson, Morgan Dufour, Aaron Zambrana, Myka Caluyong and Chris Lepage.

Contributors E-LK reviewed the manuscript after the updated search. E-LK, DBR and SK completed data extraction and risk of bias assessments. DBR, PAP, E-LK, BG and SK completed updated review of full-text articles. E-LK and RS completed the initial review of full-text articles, data extraction, risk of bias assessments and wrote the manuscript. TH codeveloped the protocol for this review and oversaw abstract screening. WC and BH provided methodological expertise in the area of network meta-analysis, conducted all statistical analyses and prepared figures. BS wrote and performed the search strategy. BG reviewed the studies and contributed to the tables describing the characteristics of CBT and MBSR studies as well as assisting in the manuscript revisions and submission. BS provided expertise as an information specialist consultant in searching for articles to review. PAP codeveloped the review protocol and oversaw all aspect of the projects. All authors contributed to manuscript writing and review. All authors read and approved the final manuscript.

Funding BH is supported by a New Investigator award from the Canadian Institutes of Health Research and the Drug Safety and Effectiveness Network. No funding was received for this study.

Competing interests None declared.

Patient consent Not required.

Provenance and peer review Not commissioned; externally peer reviewed.

Data sharing statement Any additional unpublished data from this meta-analysis will be provided upon request by the corresponding author.

\section{REFERENCES}

1 IASP. Unrelieved pain is a major global healthcare problem. 2004.

2 Morley S, Williams A, Hussain S. Estimating the clinical effectiveness of cognitive behavioural therapy in the clinic: evaluation of a CBT informed pain management programme. Pain 2008;137:670-80.

3 Ehde DM, Dillworth TM, Turner JA. Cognitive-behavioral therapy for individuals with chronic pain: efficacy, innovations, and directions for research. Am Psychol 2014:69:153-66.

4 Kabat-Zinn J, Hanh TN. Full catastrophe living: Using the wisdom of your body and mind to face stress, pain, and illness. Delta, 2009.

5 Hatchard T, Lepage C, Hutton B, et al. Comparative evaluation of group-based mindfulness-based stress reduction and cognitive behavioral therapy for the treatment and management of chronic pain disorders: protocol for a systematic review and meta-analysis with indirect comparisons. Syst Rev 2014;3:134.

6 Caldwell DM, Ades AE, Higgins JP. Simultaneous comparison of multiple treatments: combining direct and indirect evidence. BMJ 2005;331:897-900

7 Catalá-López F, Tobías A, Cameron C, et al. Network meta-analysis for comparing treatment effects of multiple interventions: an introduction. Rheumatol Int 2014;34:1489-96

8 Turk DC, Dworkin RH, Allen RR, et al. Core outcome domains for chronic pain clinical trials: IMMPACT recommendations. Pain 2003;106:337-45.

9 Hutton B, Salanti G, Caldwell DM, et al. The PRISMA extension statement for reporting of systematic reviews incorporating network meta-analyses of health care interventions: checklist and explanations. Ann Intern Med 2015;162:777.

10 Merskey H, Bogduk N. Classification of chronic pain, Part III: pain terms, a current list with definitions and notes on usage. 2nd edn, 2011.

11 Kabat-Zinn J. An outpatient program in behavioral medicine for chronic pain patients based on the practice of mindfulness meditation: theoretical considerations and preliminary results. Gen Hosp Psychiatry 1982;4:33-47.
12 Kabat-Zinn J. Full catastrophe living: using the wisdom of your body and mind to face stress pain, and illness: Delta Trade Paperbacks, 1990.

13 Downs SH, Black N. The feasibility of creating a checklist for the assessment of the methodological quality both of randomised and non-randomised studies of health care interventions. J Epidemio/ Community Health 1998;52:377-84.

14 Smeets RJ, Vlaeyen JW, Kester AD, et al. Reduction of pain catastrophizing mediates the outcome of both physical and cognitive-behavioral treatment in chronic low back pain. J Pain 2006:7:261-71.

15 National Institute for Health and Care Excellence. Evidence Synthesis TSD series NICE decision support unit. http://scharr.dept.shef.ac.uk/nicedsu/technical-supportdocuments/evidence-synthesis-tsd-series/ (Accessed 6 Sep 2017).

16 Cohen J. Statistical power analysis for the behavioral sciences. second. New York: Lawrence Erlbaum Associates, 1988

17 Spiegelhalter D, Thomas A, Best N, et al. OpenBUGS user manual. 2014 http://www. openbugs.net/w/Manuals.

18 Sturtz S, Ligges U. A package for running WinBUGS from R. J Stat Softw 2005;12 https://doaj.org/article/74babe0e462a412bafa3601cbb4a2f3a

19 DerSimonian R, Laird N. Meta-analysis in clinical trials. Control Clin Trials 1986;7:177-88

20 Alda M, Luciano JV, Andrés E, et al. Effectiveness of cognitive behaviour therapy for the treatment of catastrophisation in patients with fibromyalgia: a randomised controlled trial. Arthritis Res Ther 2011;13:R173.

21 Kraaimaat FW, Brons MR, Geenen R, et al. The effect of cognitive behavior therapy in patients with rheumatoid arthritis. Behav Res Ther 1995:33:487-95.

22 la Cour P, Petersen M. Effects of mindfulness meditation on chronic pain: a randomized controlled trial. Pain Med 2015;16:641-52.

23 Langford MM. The efficacy of a combined cognitive-behavioural and interpersonal therapy approach to the treatment of fibromyalgia syndrome : a randomized controlled trial. 2008 https://ecommons.usask.ca/handle/10388/etd-07232008113842

24 Mishra KD, Gatchel RJ, Gardea MA. The relative efficacy of three cognitivebehavioral treatment approaches to temporomandibular disorders. J Behav Med 2000:23:293-309

25 Pradhan EK, Baumgarten M, Langenberg P, et al. Effect of mindfulness-based stress reduction in rheumatoid arthritis patients. Arthritis Rheum 2007:57:1134-42.

26 Puder RS. Age analysis of cognitive-behavioral group therapy for chronic pain outpatients. Psychol Aging 1988;3:204-7.

27 Schmidt S, Grossman P, Schwarzer B, et al. Treating fibromyalgia with mindfulnessbased stress reduction: results from a 3 -armed randomized controlled trial. Pain 2011;152:361-9.

28 Sephton SE, Salmon P, Weissbecker I, et al. Mindfulness meditation alleviates depressive symptoms in women with fibromyalgia: results of a randomized clinical trial. Arthritis Rheum 2007:57:77-85.

29 Shpaner M, Kelly C, Lieberman G, et al. Unlearning chronic pain: a randomized controlled trial to investigate changes in intrinsic brain connectivity following cognitive behavioral therapy. Neuroimage Clin 2014:5:365-76.

30 Turner JA, Clancy S. Comparison of operant behavioral and cognitive-behavioral group treatment for chronic low back pain. J Consult Clin Psychol 1988;56:261-6.

31 Cash E, Salmon P, Weissbecker I, et al. Mindfulness meditation alleviates fibromyalgia symptoms in women: results of a randomized clinical trial. Ann Behav Med 2015:49:319-30

32 Vallejo MA, Ortega J, Rivera J, et al. Internet versus face-to-face group cognitivebehavioral therapy for fibromyalgia: a randomized control trial. J Psychiatr Res 2015;68:106-13.

33 Castel A, Salvat M, Sala J, et al. Cognitive-behavioural group treatment with hypnosis: a randomized pilot trail in fibromyalgia. Contemporary Hypnosis 2009;26:48-59.

34 Cherkin DC, Sherman KJ, Balderson BH, et al. effect of mindfulness-based stress reduction vs cognitive behavioral therapy or usual care on back pain and functional limitations in adults with chronic low back pain: a randomized clinical trial. JAMA 2016;315:1240.

35 Esmer G, Blum J, Rulf J, et al. Mindfulness-based stress reduction for failed back surgery syndrome: a randomized controlled trial. J Am Osteopath Assoc 2010;110:646-52

36 Falcão DM, Sales L, Leite JR, et al. Cognitive behavioral therapy for the treatment of fibromyalgia syndrome: a randomized controlled trial. J Musculoskelet Pain 2008; 16:133-40.

37 Jensen $\mathrm{KB}$, Kosek E, Wicksell $\mathrm{R}$, et al. Cognitive behavioral therapy increases pain-evoked activation of the prefrontal cortex in patients with fibromyalgia. Pain 2012; 153:1495-503

38 Kearney DJ, Simpson TL, Malte CA, et al. Mindfulness-based stress reduction in addition to usual care is associated with improvements in pain, fatigue, and cognitive failures among veterans with gulf war illness. Am J Med 2016;129:204-14.

39 Keefe FJ, Caldwell DS, Williams DA, et al. Pain coping skills training in the management of osteoarthritic knee pain: a comparative study. Behav Ther 1990;21:49-62.

40 Veehof MM, Trompetter HR, Bohlmeijer ET, et al. Acceptance- and mindfulness-based interventions for the treatment of chronic pain: a meta-analytic review. Cogn Behav Ther 2016;45:5-31 
41 Williams ACdeC, Eccleston C, Morley S. Psychological therapies for the management of chronic pain (excluding headache) in adults. Cochrane Database Syst Rev; 19.

42 Castonguay LG. Change in Psychotherapy: a plea for no more "Nonspecific" and False dichotomies. Clinical Psychology: Science and Practice 2005;12:198-201.

43 Waltz J, Addis ME, Koerner K, et al. Testing the integrity of a psychotherapy protocol: assessment of adherence and competence. J Consult Clin Psychol 1993;61:620-30.
44 Moncher FJ, Prinz RJ. Treatment fidelity in outcome studies. Clin Psychol Rev 1991;11:247-66.

45 Brauhardt A, de Zwaan M, Herpertz S, et al. Therapist adherence in individual cognitive-behavioral therapy for binge-eating disorder: assessment, course, and predictors. Behav Res Ther 2014;61:55-60.

46 Vlaeyen JWS, Morley S. Cognitive-behavioral treatments for chronic pain. : What works for whom? 2005:21: 1-8. 